\title{
Self and mutual magnetic helicities in coronal magnetic configurations
}

\author{
S. Régnier ${ }^{1}$, T. Amari ${ }^{2}$, and R. C. Canfield ${ }^{3}$ \\ 1 ESA Research and Scientific Support Department, ESTEC, Keplerlaan 1, 2201 AZ Noordwijk, The Netherlands \\ e-mail: sregnier@rssd.esa.int \\ 2 Centre de Physique Théorique, École Polytechnique, 91128 Palaiseau, France \\ 3 Montana State University, Physics Dept., 264 EPS Building, Bozeman, MT 59717, USA
}

Received 24 May 2005 / Accepted 4 July 2005

\section{ABSTRACT}

Together with the magnetic energy, the magnetic helicity is an important quantity used to describe the nature of a magnetic field configuration. In the following, we propose a new technique to evaluate various components of the total magnetic helicity in the corona for an equilibrium reconstructed magnetic field. The most meaningful value of helicity is the total relative magnetic helicity which describes the linkage of the field lines even if the volume of interest is not bounded by a magnetic surface. In addition if the magnetic field can be decomposed into the sum of a closed field and a reference field (following Berger 1999, in Magnetic Helicity in Space and Laboratory Plasmas, ed. M. R. Brown, R. C. Canfield, \& A. A. Pevtsov, 1), we can introduce three other helicity components: the self helicity of the closed field, the mutual helicity between the closed field and the reference field, and the vacuum helicity (self helicity of the reference field). To understand the meaning of those quantities, we derive them from the potential field (reference) and the force-free field computed with the same boundary conditions for three different cases: a single twisted flux tube derived from the extended Gold-Hoyle solutions, a simple magnetic configuration with three balanced sources and a constant distribution of the force-free parameter, and the AR 8210 magnetic field observed from 17:13 UT to 21:16 UT on May 1,1998 . We analyse the meaning of the self and mutual helicities: the self and mutual helicities correspond to the twist and writhe of confined flux bundles, and the crossing of field lines in the magnetic configuration respectively. The main result is that the magnetic configuration of AR 8210 is dominated by the mutual helicity and not by the self helicity (twist and writhe). Our results also show that although not gauge invariant the vacuum helicity is sensitive to the topological complexity of the reference field.

Key words. Sun: magnetic fields - Sun: corona - Sun: flares

\section{Introduction}

To understand how the magnetic energy is stored in a magnetic configuration and how the magnetic field lines are linked, one relevant quantity is the magnetic helicity, which characterises the twist, writhe and linkage of field lines. Due to those properties, the estimate of the helicity content inside an active region has became an important topic in recent years (see Brown \& Priest 1999). The magnetic helicity in the corona cannot be directly derived from observations. Recently, Chae et al. (2001) developed a method for determining the helicity flux through the photosphere using photospheric magnetic field measurements. The time evolution of the magnetic helicity on the photosphere characterizes both the injection of magnetic helicity from the convection zone into the solar atmosphere, and the changes in the coronal magnetic field configurations related to eruptive events propagating into the interplanetary medium like coronal mass ejections. Pre-flare and post-flare relative budget of a flaring active region (Bleybel et al. 2002) are thus also instructive. It is worth noticing that this does not necessary imply the existence of bounds on magnetic helicity which could be the evidence for the trigger of an eruptive event, since it has been shown than constant helicity mechanisms can lead to such disruptions (Amari et al. 2003a,b). Several methods have been developed to estimate the magnetic helicity injection rate from photospheric magnetic field measurements (line-of-sight or vector measurements) and for which the flow field on the photosphere is needed (Chae et al. 2001; Démoulin et al. 2002; Nindos \& Zhang 2002; Kusano et al. 2002; Moon et al. 2002; Démoulin et al. 2003; Chae et al. 2004). In this Paper, we have a different approach to compute the magnetic helicity and its evolution in the corona: we use the magnetic field vector and the vector potential in the coronal half-space above the photospheric plane of measurement, determined by a force-free extrapolation method. Those two vectors allow us to directly evaluate the relevant magnetic helicity. It is worth noticing that unlike other previous time estimates of magnetic helicity, in the present Paper one assumes that the configuration always evolves through a series of equilibria.

To understand the meaning of different helicity calculations (see Sect. 2), we use two different approaches: we first study the helicity content of force-free solutions with twisted field lines or topological elements (e.g. null points, spine field lines, fan 
surfaces), and secondly we apply this method to a solar active region. We also follow the time evolution of the magnetic helicities and their link to the flaring activity of the active region.

\section{Magnetic helicities}

The magnetic helicity describes the complexity of a magnetic configuration in terms of topology and of linkage of field lines:

$H_{\mathrm{m}}(\boldsymbol{B})=\int_{V} \boldsymbol{A} \cdot \boldsymbol{B} \mathrm{d} V$

where $\boldsymbol{B}$ and $\boldsymbol{A}$ describe the magnetic field in the volume $V$. The helicity value depends on the gauge condition imposed on the vector potential $\boldsymbol{A}$. Nevertheless a gauge-free quantity is defined as the relative magnetic helicity (helicity of the field $\boldsymbol{B}$ relative to a reference field):

$\Delta H_{\mathrm{m}}\left(\boldsymbol{B}, \boldsymbol{B}_{\mathrm{pot}}\right)=\int_{V}\left(\boldsymbol{A}+\boldsymbol{A}_{\mathrm{pot}}\right) \cdot\left(\boldsymbol{B}-\boldsymbol{B}_{\mathrm{pot}}\right) \mathrm{d} V$

given by Finn \& Antonsen (1985). $\boldsymbol{B}$ and $\boldsymbol{A}$ here describe the nonlinear force-free ( $n l f f$ ) field, $\boldsymbol{B}_{\text {pot }}$ and $\boldsymbol{A}_{\text {pot }}$ represent the potential field used as a reference field. In addition, we derive other relevant helicities. Following Berger (1999), we decompose the magnetic field into two fields:

$\boldsymbol{B}=\boldsymbol{B}_{\mathrm{cl}}+\boldsymbol{B}_{\text {pot }}$

where $\boldsymbol{B}_{\mathrm{cl}}$ is a closed field with no flux through the boundaries, the reference field $\boldsymbol{B}_{\text {pot }}$ satisfies the following properties:

$\boldsymbol{\nabla} \wedge \boldsymbol{B}_{\text {pot }}=\mathbf{0}$,

and the magnetic field normal to the boundaries is the same as for $\boldsymbol{B}$ :

$\boldsymbol{B}_{\mathrm{n}}=\boldsymbol{B}_{\mathrm{n}, \text { phot }}=g$

where $g$ is the distribution of the magnetic field on the photosphere (or at the lower boundary) and

$\boldsymbol{B}_{\mathrm{n}}=\boldsymbol{B}_{\mathrm{n}, \text { phot }}=\mathbf{0}$

on the other sides of the computational box. Then two kinds of helicity are defined (Berger 1999; Priest 1999): the self helicity $H_{\text {self }}$ which is computed for a given magnetic field $\left(\boldsymbol{B}, \boldsymbol{B}_{\mathrm{cl}}\right.$ or $\boldsymbol{B}_{\text {pot }}$ ), and the mutual helicity $H_{\text {mut }}$ between two different fields:

$H_{\text {self }}(\boldsymbol{B})=\int_{V} \boldsymbol{A} \cdot \boldsymbol{B} \mathrm{d} V=H_{\mathrm{m}}(\boldsymbol{B})$

and

$H_{\text {mut }}\left(\boldsymbol{B}_{\text {pot }}, \boldsymbol{B}_{\mathrm{cl}}\right)=2 \int_{V} \boldsymbol{A}_{\text {pot }} \cdot \boldsymbol{B}_{\mathrm{cl}} \mathrm{d} V$.

Equations (7) and (8) are computed using the nlff field and the potential field in the volume above the photosphere. Equation (7) is applied to both closed and potential fields. As noticed by Berger (1999), the sum of the self helicity of the closed field and the mutual helicity is the relative magnetic helicity given by Eq. (2):

$$
\begin{aligned}
\Delta H_{\mathrm{m}}\left(\boldsymbol{B}, \boldsymbol{B}_{\mathrm{pot}}\right) & =H_{\mathrm{m}}(\boldsymbol{B})-H_{\mathrm{m}}\left(\boldsymbol{B}_{\mathrm{pot}}\right) \\
& =H_{\mathrm{self}}\left(\boldsymbol{B}_{\mathrm{cl}}\right)+H_{\mathrm{mut}}\left(\boldsymbol{B}_{\mathrm{pot}}, \boldsymbol{B}_{\mathrm{cl}}\right) .
\end{aligned}
$$

Berger (1999) mentions that the reference field is a minimum energy state and then a zero energy density can be assigned to this field. It is therefore natural to impose a zero helicity for the vacuum field in a simply connected domain.

In Berger (1988), the self helicity of the potential field is zero for the following conditions on $\boldsymbol{A}_{\text {pot }}$ :

$\boldsymbol{\nabla} \wedge \boldsymbol{A}_{\mathrm{pot}}=\boldsymbol{B}_{\mathrm{pot}}, \quad \boldsymbol{\nabla} \cdot \boldsymbol{A}_{\mathrm{pot}}=0, \quad \boldsymbol{A}_{\mathrm{pot}} \cdot \boldsymbol{n}_{\mid S}=0$

where $S$ is boundary surface of the coronal volume. In practice, we compute the force-free field using the XTRAPOL code as described in Amari et al. (1997). The vector potential and the magnetic field are computed using a Grad-Rubin-like method used to determine the solution of the nonlinear force-free equilibrium equations. We then easily compute the various magnetic helicities defined above.

The last condition of Eq. (10) is replaced by $\partial_{\mathrm{n}} \boldsymbol{A}_{\text {pot }}=\mathbf{0}$ owing to the different gauge condition used by the authors. Therefore with those boundary conditions, the self helicity of the vacuum field is not zero in the coronal volume.

Equation (2) from Finn \& Antonsen (1985) can be directly derived from Eq. (9). In 1984, Berger \& Field have derived another formula for the relative helicity given by

$\Delta H_{\mathrm{m}}=\int_{V}\left(\boldsymbol{A}-\boldsymbol{A}_{\mathrm{pot}}\right) \cdot\left(\boldsymbol{B}+\boldsymbol{B}_{\mathrm{pot}}\right) \mathrm{d} V$

which is equal to the Finn \& Antonsen formula when the halfspace above the photosphere is considered.

Helicity estimates provided in Bleybel et al. (2002) have been performed using this formulae. It is important to note that the otherwise remaining contributing term (due to finite domain) has been shown to vanish for boundary conditions Eq. (6) or negligible for boundaries put far enough.

\section{Examples}

We now describe the results obtained by computing the self helicity (Eq. (7) applied to the closed field), the mutual helicity, the relative helicity from Eq. (2) and the vacuum helicity (self helicity of the potential field) for several examples: four extended Gold \& Hoyle solutions representing a single twisted flux tube, two linear force-free field solutions associated with the distribution of three balanced sources on the photosphere, and a time series of nonlinear force-free configurations associated with the AR 8210 observed on May 1, 1998.

\subsection{Extended Gold-Hoyle solutions}

The Extended Gold-Hoyle (EG\&H) solutions (Régnier 2005) describe nonlinear force-free configurations for non-uniformly twisted flux tubes. We use the EG\&H solutions as bottom boundary condition given by the following expressions:

$B_{z}(r)=\frac{B_{0}}{\left(1+\kappa \frac{r^{2}}{r_{0}^{2}}\right)^{\kappa}}$,

$B_{\theta}(r)=\frac{B_{0} \frac{r_{0}}{r}}{\sqrt{\kappa(2 \kappa-1)}}\left[1-\frac{1+2 \kappa^{2} \frac{r^{2}}{r_{0}^{2}}}{\left(1+\kappa \frac{r^{2}}{r_{0}^{2}}\right)^{2 \kappa}}\right]^{\frac{1}{2}}$ 


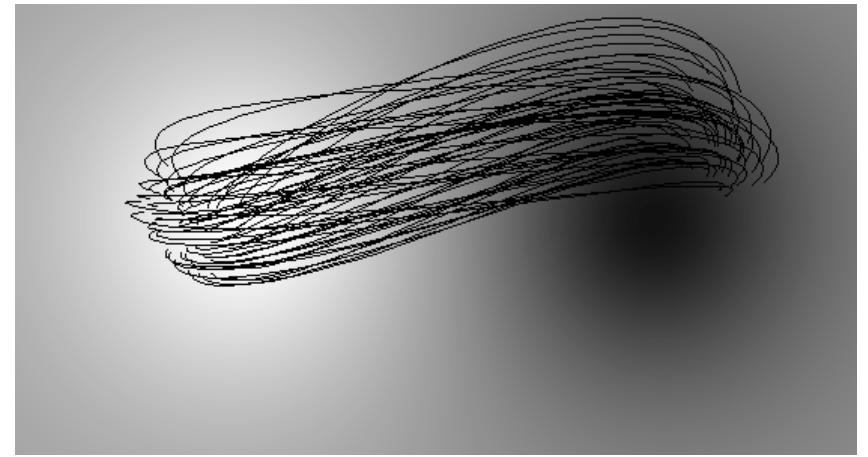

Fig. 1. EG\&H solution for $\kappa=2$ and $r_{0}=1$. Only few field lines are plotted for the sake of clarity.

and

$$
\alpha(r)=\frac{2 \kappa^{2} \sqrt{\kappa(2 \kappa-1)} r^{2}}{r_{0}^{3}\left(1+\kappa \frac{r^{2}}{r_{0}^{2}}\right)\left[\left(1+\kappa \frac{r^{2}}{r_{0}^{2}}\right)^{2 \kappa}-\left(1+2 \kappa^{2} \frac{r^{2}}{r_{0}^{2}}\right)\right]^{\frac{1}{2}}}
$$

where $r$ is the distance from the center of a polarity. We use a simple dipolar magnetic configuration with a characteristic length of $100 \mathrm{Mm}$ and a magnetic field strength of $2000 \mathrm{G}$ for 4 sets of parameters $\left(\kappa, r_{0}\right):(0.8,0.56),(0.8,0.4),(2,1.4),(2,1)$. Figure 1 is an example of a nonuniformly twisted flux tube reconstructed under the nlff assumption using $B_{z}$ from Eq. (12) and $\alpha$ from Eq. (14) in only one polarity on the lower boundary surface ("photosphere"). To evaluate the helicities, the volume $V$ is $100 \times 100 \times 100 \mathrm{Mm}$.

\subsection{Magnetic configurations with three sources}

We define the photospheric distribution of the vertical magnetic field for a three-source configuration: two negative polarities N1 and N2, and one positive polarity P1 (see Brown \& Priest 1999). $B_{z}$ is given by a Gaussian distribution:

$B_{z}(r)=B_{0} \exp \left(-\frac{r^{2}}{\sigma^{2}}\right)$

where $r$ is radius from the center of the polarity, $\sigma$ is the width of the Gaussian ( $\sigma=0.03$ for each polarity). The magnetic field strengths are: $B_{0, P 1}=2000 \mathrm{G}, B_{0, N 1}=-1333.33 \mathrm{G}$ and $B_{0, N 2}=-666.66 \mathrm{G}$. The angle between the two negative polarities relative to the positive polarity is $90^{\circ}$. The total magnetic flux on the photosphere is balanced. The force-free parameter $\alpha$ is assumed to be a constant everywhere. We have choosen two values of $\alpha\left(0.04\right.$ and $\left.0.08 \mathrm{Mm}^{-1}\right)$. The skeleton (set of topological elements) of the magnetic configurations contains a negative null point (triangle), a spine field line (thick white line) and a fan (thin black line) as described in Fig. 2. The volume $V$ is $150 \times 150 \times 100 \mathrm{Mm}$.

\section{3. $A R 8210$}

AR 8210 was observed on May 1, 1998 and was the site of numerous flares. We have a time series of 15 averaged vector magnetograms recorded by IVM (Imaging Vector Magnetograph, Mickey et al. 1996). From 17:00 UT to



Fig. 2. Magnetic configuration with 3 sources for $\alpha=0.08 \mathrm{Mm}^{-1}$. The topological elements are defined: negative null point (triangle), the spine (white thick line) and the fan (black line). The third eigenvalue is perpendicular to the photospheric plane.

21:40 UT, we determine the 3D coronal magnetic field evolution assuming a nlff equilibrium at each time of observation. The spatial resolution of the IVM (1". 1 pixels were used in May 1998) does not allow us to fully resolve the transverse field (or the electric current density) of low-lying structures (e.g., H $\alpha$ filaments). As argued by Parker (1996), small scale electric currents, which cannot be resolved by current instruments, almost certainly exist on the Sun. We have no way of estimating the spatial scales of such currents, and hence the effects on our observations. A complete study of the topology and the flare processes of AR 8210 has been done in Régnier \& Canfield (2004, 2005). In Fig. 3, the complex topology of AR 8210 including null points and separatrix surfaces is evidenced by the changes in connectivity of the plotted field lines. During the time period of interest, the main photospheric motion (Welsch et al. 2004; Longcope 2004) is the clockwise rotation of the sunspot associated with a southward displacement of the positive polarity located on the South-East side of the sunspot (Régnier \& Canfield 2004). The coronal volume $V$ is $430 \times 325 \times 335 \mathrm{Mm}$.

\section{Discussion and conclusions}

We have evaluated the relative, self, mutual and vacuum helicities in the corona based on the magnetic field vector and the associated vector potential derived from a nlff extrapolation (Amari et al. 1997). It is worth noticing that the results depend on the assumption made on the nature of the magnetic field in the corona. As an example, Régnier et al. (2003) have estimated the relative magnetic helicity for linear force-free fields by varying $\alpha$ and for the nonlinear force-free assumption. The authors performed the computations with the same boundary conditions to be able to compare their results. The main 
Table 1. Magnetic helicities for different models (unit of $10^{43} \mathrm{G}^{2} \mathrm{~cm}^{4}$ ): 4 EG\&H solutions (see text for details), 2 linear force-free solutions for three sources distribution on the photosphere $\left(\alpha\right.$ unit is $\left.\mathrm{Mm}^{-1}\right)$, and the $n l f f$ field for AR 8210 at 19:40 UT. The percentage in parenthesis is computed with respect to the relative helicity. The last two columns give $H_{\text {obs }}$ values from Eq. (16) for $\alpha_{\text {mean }}$ and $\alpha_{\text {peak }}$ (see text for details).

\begin{tabular}{|c|c|c|c|c|c|c|}
\hline Model & $\begin{array}{c}\text { Self helicity } \\
H_{\text {self }}\left(\boldsymbol{B}_{\mathrm{cl}}\right)\end{array}$ & $\begin{array}{c}\text { Mutual helicity } \\
H_{\text {mut }}\left(\boldsymbol{B}_{\text {pot }}, \boldsymbol{B}_{\mathrm{cl}}\right)\end{array}$ & $\begin{array}{c}\text { Relative helicity } \\
\Delta H_{\mathrm{m}}\left(\boldsymbol{B}, \boldsymbol{B}_{\mathrm{pot}}\right)\end{array}$ & $\begin{array}{c}\text { Vacuum helicity } \\
H_{\text {self }}\left(\boldsymbol{B}_{\text {pot }}\right)\end{array}$ & $\begin{array}{l}H_{\text {obs }} \\
\alpha_{\text {mean }}\end{array}$ & $\begin{array}{l}H_{\text {obs }} \\
\alpha_{\text {peak }} \\
\end{array}$ \\
\hline EG\&H solutions & & & & & & \\
\hline$\kappa=0.8, r_{0}=0.56$ & $0.13(2.2 \%)$ & $5.6(96 \%)$ & 5.8 & $-3.7 \times 10^{-4}\left(6 \times 10^{-3} \%\right)$ & 12.6 & 35.7 \\
\hline$\kappa=0.8, r_{0}=0.4$ & $0.38(4.4 \%)$ & $8.2(96 \%)$ & 8.5 & $-3.7 \times 10^{-4}\left(4 \times 10^{-3} \%\right)$ & 17.6 & 50.1 \\
\hline$\kappa=2, r_{0}=1.4$ & $0.15(2.9 \%)$ & $4.8(95 \%)$ & 5.1 & $-3.4 \times 10^{-4}\left(7 \times 10^{-3} \%\right)$ & 11.3 & 26.2 \\
\hline$\kappa=2, r_{0}=1$ & $0.44(5.8 \%)$ & $7.2(94 \%)$ & 7.6 & $-3.4 \times 10^{-4}\left(4 \times 10^{-3} \%\right)$ & 15.8 & 36.6 \\
\hline $\begin{array}{c}\text { Trisources } \\
\alpha=0.04\end{array}$ & $7.92 \times 10^{-5}(34 \%)$ & $9.08 \times 10^{-5}(62 \%)$ & $1.45 \times 10^{-4}$ & $4.9 \times 10^{-5}(33 \%)$ & \multirow{2}{*}{\multicolumn{2}{|c|}{$\begin{array}{l}4.68 \times 10^{-2} \\
9.37 \times 10^{-2}\end{array}$}} \\
\hline$\alpha=0.08$ & $1.24 \times 10^{-3}(86 \%)$ & $2.44 \times 10^{-4}(16 \%)$ & $1.44 \times 10^{-3}$ & $4.9 \times 10^{-5}(3.5 \%)$ & & \\
\hline $\begin{array}{c}\text { AR } 8210 \text { on May } 1,1998 \\
\text { at } 19: 40 \text { UT }\end{array}$ & $-8.8 \times 10^{-2}(21 \%)$ & $-0.36(86 \%)$ & -0.42 & $2 \times 10^{-2}(4.7 \%)$ & 1.67 & -32.8 \\
\hline
\end{tabular}



Fig. 3. Characteristic flux bundles describing the magnetic configuration of AR 8210 on May 1, 1998 at 19:40 UT. We notice the complex topology of the field evidenced by connectivity changes from one flux bundle to an other.

conclusion is that the relative helicity computed for the linear force-free field can be very different in absolute value and even in sign from the relative helicity computed for the nlff field.

In Table 1, we summarize the different helicity values computed for the three examples. The results are discussed below.

For the reconstructed EG\&H solutions, the main contribution to the relative helicity is the mutual helicity $(\sim 95 \%)$. The vacuum helicity value is negligible and is opposite in sign to the other helicities. The mutual helicity is constant when the twist increases (increasing $r_{0}$ ). When $\alpha_{0}\left(=\frac{\kappa}{r_{0}}\right)$ increases by a factor of 1.4, the self helicity increases by a factor of 3 and the contribution to the relative helicity increases by a factor of 2 . The relative magnetic helicity increases by $\sim 47 \%$ when $\alpha_{0}$ is multiplied by 1.4 . The EG\&H solutions describe nonuniformly twisted flux tubes. Therefore it is surprising that the helicity content is dominated by the mutual helicity and not the self helicity characterizing the twist of a flux tube. The fact is the nonuniformly twisted flux tube fills the entire volume: the flux tube is not confined. Therefore the twist is a large scale twist (see Fig. 1) which can be seen as the crossing of the field lines, i.e. a mutual helicity. Following Leamon et al. (2004) and
Moffatt \& Ricca (1992), a good estimate of the helicity related to the twist from observations is given by

$H_{\mathrm{obs}}=\frac{1}{8} \Phi^{2} \alpha L$

where $\Phi$ is the total unsigned magnetic flux on the photosphere, $L$ is a characteristic length of the active region (e.g., length between the center of mass of each polarity). In Table 1 we compute $H_{\mathrm{obs}}$ for the differents models and for $\alpha$ defined as the mean $\alpha$ value, or the $\alpha_{\text {peak }}$ defined by Leka et al. (2005). To apply Eq. (16) we assume that the studied magnetic configurations correspond to a single twisted flux tube. For the EG\&H solutions, the total unsigned flux (Régnier 2005) is $4.5 \times 10^{22} \mathrm{Gcm}^{2}$ for $\kappa=0.8$ and $4.28 \times 10^{22} \mathrm{Gcm}^{2}$ for $\kappa=2$. The mean $\alpha$ values are $1.25,1.75,1.23$ and 1.73 (unit of $10^{-2} \mathrm{Mm}^{-1}$ ) for the four cases. $\alpha_{\text {peak }}$ is simply $\alpha_{0}$. The characteristic length $L=40 \mathrm{Mm}$ is the distance between the centers of each polarity. For the three-source case, the unsigned flux is $2.5 \times 10^{21} \mathrm{G} \mathrm{cm}^{2}$. The characteristic length $L=15 \mathrm{Mm}$ is the distance between the centers of mass of the negative and positive polarities. For AR 8210, the unsigned flux is $2.56 \times 10^{22} \mathrm{G} \mathrm{cm}^{2}, \alpha_{\text {mean }}$ is $6.1 \times 10^{-3} \mathrm{Mm}^{-1}$ and $\alpha_{\text {peak }}$ is $-0.12 \mathrm{Mm}^{-1}$ and the characteristic length is $45 \mathrm{Mm}$. In every case, $H_{\text {obs }}$ overestimates the relative magnetic helicity. In the case of a single flux tube described by the EG\&H solutions and for which Eq. (16) is the most suitable, $H_{\mathrm{obs}}$ is a factor 2-6 above $\Delta H_{\mathrm{m}}$. For the three-source case and AR 8210, there is a factor of 4-400 difference between $H_{\mathrm{obs}}$ and $\Delta H_{\mathrm{m}}$. Therefore, Eq. (16) give a reasonable value of the magnetic helicity only when a single flux tube is considered. Equation (16) has to be applied with caution to solar active region as Leamon et al. (2004) have done.

For the linear force-free field configurations with three sources, the self and mutual helicities both have an important contribution to the relative helicity depending on the value of $\alpha$ inside the configuration. The vacuum helicity is not negligible but has a contribution that decreases rapidly when $\alpha$ increases. The relative helicity is multiplied by a factor of 10 when $\alpha$ is doubled.

In Fig. 4, we plot the time evolution of the relative magnetic helicity given by the Finn \& Antonsen formula (solid line), the mutual helicity between potential and close fields (dashed 


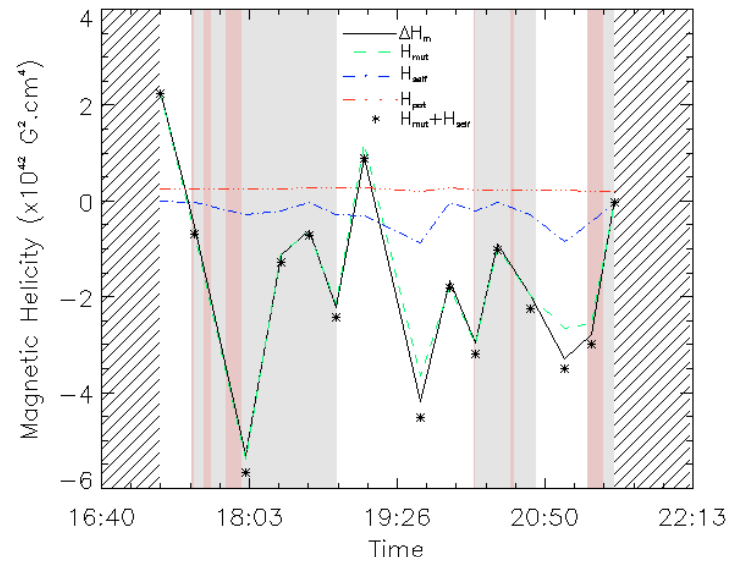

Fig. 4. Time evolution of magnetic helicities (unit of $10^{42} \mathrm{G}^{2} \mathrm{~cm}^{4}$ ) as $H_{\text {self }}\left(\boldsymbol{B}_{\mathrm{cl}}\right)$ (dot-dashed line), $H_{\text {mut }}\left(\boldsymbol{B}_{\mathrm{pot}}, \boldsymbol{B}_{\mathrm{cl}}\right)$ (dashed line), $H_{\mathrm{m}}\left(\boldsymbol{B}_{\mathrm{pot}}\right)$ or vacuum helicity (dot-dot-dot-dashed line), $\Delta H_{\mathrm{m}}$ from the FinnAntonsen formula (solid line) and the sum of self and mutual helicity (stars). Gray areas are the flaring periods, dark gray areas are the impulsive phase of flares.

line), the self helicity of the close field (dot-dashed line), the vacuum helicity (dot-dot-dot-dashed line) and the sum of the mutual helicity and the self helicity (stars). As expected from Eq. (9), the sum of the self and mutual helicities (stars) follows the evolution of the relative magnetic helicity (solid line). The mutual helicity is the main contribution to the relative magnetic helicity of AR 8210 which means that the way the field lines crossing between each other is the essential part of the helicity and not the twist or the writhe inside AR 8210. The self helicity of AR 8210 stays relatively small $\left(\sim-10^{42} \mathrm{G}^{2} \mathrm{~cm}^{4}\right)$ throughout the time period shown in the figure. this implies that neither the twist nor writhe of closed flux bundles (e.g., twist in filaments and complex topology of separatrix surfaces seen in Sects. 3.2 and 3.3) varies much during this period. It is clear from Fig. 3 that it is over-simplified to interpret the self-helicity of this region in terms of a single twisted flux tube emerging from the convection zone, as other authors have done for other ARs. However, if we do so, using Eq. (16), and the thin-flux tube relationship ( $\alpha=2 q$, Longcope et al. 1998) to relate $\alpha$ to the pitch of the magnetic field, we conclude that the equivalent number of turns $\mathrm{qL}$ is only about $10^{-2}$. We note that the helicity of the vacuum field is a constant with a positive value of $\sim 2 \times 10^{41} \mathrm{G}^{2} \mathrm{~cm}^{4}$. The uncertainties on the measurement of the magnetic helicity cannot be derived directly from the computed quantities. Nevertheless we have evaluated the errors on the computation of the magnetic energy from the virial theorem (Klimchuk et al. 1992). Applying the Klimchuk et al. method, we estimate that the errors are less than $1 \%$.

The meaning of the self and mutual helicities can easily be understood: the self helicity characterises the twist and the writhe of confined flux bundles, the mutual helicity characterises the crossing of field lines which also includes large scale twist. The vacuum helicity is more difficult to interpret because this quantity is not gauge invariant. From this study of both theoretical and observational cases, it appears that a small amount of vacuum helicity ( $>1 \%$ of the relative helicity) exists in a magnetic configuration with topological elements.
Acknowledgements. We would like to thank M. Berger and the referee for fruitful comments on the manuscript. S. R. research is funded by the European Commission's Human Potential Programme throught the European Solar Magnetism Network (contract HPRN-CT-200200313). The computation have been performed with the AFOSR support under the DoD MURI grant, "Understanding Solar Eruptions and their Interplanetary Consequences".

\section{References}

Amari, T., Aly, J. J., Luciani, J.-F., Boulmezaoud, T. Z., \& Mikic, Z. 1997, Sol. Phys., 174, 129

Amari, T., Luciani, J.-F., Aly, J.-J., Mikic, Z., \& Linker, J. 2003a, ApJ, 585,1073

Amari, T., Luciani, J.-F., Aly, J.-J., Mikic, Z., \& Linker, J. 2003b, ApJ, 595,1231

Berger, M. A. 1988, A\&A, 201, 355

Berger, M. A. 1999, in Magnetic Helicity in Space and Laboratory Plasmas, ed. M. R. Brown, R. C. Canfield, \& A. A. Pevtsov, 1

Berger, M. A., \& Field, G. B. 1984, Journal of Fluid Mechanics, 147, 133

Bleybel, A., Amari, T., van Driel-Gesztelyi, L., \& Leka, K. D. 2002, A\&A, 395, 685

Brown, M. R., Canfield, R. C., \& Pevtsov, A. A. 1999, in Magnetic Helicity in Space and Laboratory Plasma

Brown, D. S., \& Priest, E. R. 1999, Proc. of the Royal Soc., 455, 3931

Chae, J., Wang, H., Qiu, J., Goode, P. R., Strous, L., \& Yun, H. S. 2001, ApJ, 560, 476

Chae, J., Moon, Y., \& Park, Y. 2004, Sol. Phys., 223, 39

Démoulin, P., \& Berger, M. A. 2003, Sol. Phys., 215, 203

Démoulin, P., Mandrini, C. H., Van Driel-Gesztelyi, L., Lopez-Fuentes, M. C., \& Aulanier, G. 2002, Sol. Phys., 207, 87

Finn, J. M., \& Antonsen, T. M. 1985, Comments Plasma Phys. Controlled Fusion, 9, 111

Klimchuk, J. A., Canfield, R. C., \& Rhoads, J. E. 1992, ApJ, 385, 327

Kusano, K., Maeshiro, T., Yokoyama, T., \& Sakurai, T. 2002, ApJ, 577,501

Leamon, R. J., Canfield, R. C., Jones, S. L., et al. 2004, JGR, 109, A18, 5106

Leka, K. D., Fan, Y., \& Barnes, G. 2005, ApJ, 626, 1091

Leka, K. D., \& Skumanich, A. 1999, Sol. Phys., 188, 3

Longcope, D. W. 2004, ApJ, 612, 1181

Longcope, D. W., Fisher, G. H., \& Pevtsov, A. A. 1998, ApJ, 507, 417

Mickey, D. L., Canfield, R. C., LaBonte, B. J., et al. 1996, Sol. Phys., 168,229

Moffatt, H. K., \& Ricca, R. L. 1992, Proc. R. Soc. Lond. A, 439, 411

Moon, Y.-J., Chae, J., Wang, H., Choe, G. S., \& Park, Y. D. 2002, ApJ, 580,528

Nindos, A., \& Zhang, H. 2002, ApJ, 573, L133

Parker, E. N. 1996, ApJ, 471, 485

Pevtsov, A. A., Canfield, R. C., Metcalf, T. R. 1995, ApJ, 440, L109

Priest, E. R. 1999, in Magnetic Helicity in Space and Laboratory Plasmas, ed. M. R. Brown, R. C. Canfield, A. A. Pevtsov, 141

Régnier, S. 2005, A\&A, submitted

Régnier, S., \& Canfield, R. C. 2004, in SOHO 15: Coronal Heating, ed. R. W. Walsh, J. Ireland, D. Danesy, B. Fleck, ESA-SP 575

Régnier, S., \& Canfield, R. C. 2005, in preparation

Régnier, S., Canfield, R. C., \& Amari, T. 2003, AAS/Solar Physics Division Meeting, 34, 01.03

Welsch, B. T., Fisher, G. H., Abbett, W. P., \& Régnier, S. 2004, ApJ, 610,1148 